\title{
A Two-Station Passive Locating Solution Independent of the Baseline Length
}

\author{
Tao Yu \\ China Academy of Management Science, Beijing, China \\ Email: tyt0803@163.com
}

How to cite this paper: Yu, T. (2020) A Two-Station Passive Locating Solution Independent of the Baseline Length. Open Journal of Applied Sciences, 10, 476-485. https://doi.org/10.4236/ojapps.2020.107033

Received: May 2, 2020

Accepted: July 25, 2020

Published: July 28, 2020

Copyright $\odot 2020$ by author(s) and Scientific Research Publishing Inc. This work is licensed under the Creative Commons Attribution International License (CC BY 4.0).

http://creativecommons.org/licenses/by/4.0/

\begin{abstract}
The two-station positioning system based on time difference and azimuth measurement has measurement redundancy. Therefore, not only can a positioning solution which is completely independent of the baseline length between two stations be derived, but also the baseline length can be solved as an unknown quantity. These findings not only enhance the performance of the two-station positioning system, but also provide a design basis for the construction of a self-organizing dynamic intelligent positioning system.
\end{abstract}

\section{Keywords}

Positioning without Baseline, Two-Station Locating, Time Difference, Direction Finding, Path Difference, Passive Location, Self-Organizing System

\section{Introduction}

The direction-finding system for positioning uses multiple detection stations at different positions to detect the same radiation source, and determines the position of the radiation source through the crossing of the beam [1] [2]. For real targets, in general, the change of direction angle is slow and the range is small, which is one of the reliable parameters for detecting radiation sources. Especially in the modern dense complex signal environment, the direction parameter almost becomes a reliable radiation source parameter. In addition, the time uniformity between the detection platforms is less required when the orientation angle is used. Because of its simple structure, easy implementation and low cost, the direction-finding system has been widely used.

However, the main disadvantage of the direction-finding method lies in the large positioning error. As an improvement, a joint location method of azimuth and time difference is proposed. The most commonly used method is the joint positioning method based on azimuth and time difference measurement for two 
stations [3] [4] [5]. It realizes single positioning through two stations and combines the advantages of simple equipment of direction finding system and high precision of time difference positioning. It avoids the disadvantages of low accuracy of direction finding, large number of time difference positioning platforms and high requirement on time synchronization.

In terms of the construction mode, the joint positioning method based on the measurement of azimuth and time difference is to increase the time difference measurement on the basis of the two-station direction-finding method, so it has the redundancy of measurement in fact. Based on this redundancy, a two-station passive localization solution independent of the baseline length is given in a fairly simple mathematical way. At the same time, the formula of calculating the baseline length between two stations based on angle and time difference is also given. The former result shows that the dual-station positioning system of mixed measurement has dynamic expansion, while the latter result means that the dualstation positioning system of mixed measurement has self-restraint.

Dual station passive location without baseline may be a new concept proposed by the authors. The positioning solution of the existing multi-station positioning system is directly related to the baseline length, which reflects in the physical level that the distance between stations must be determined when the stations are deployed. The positioning method without baseline can further enhance the engineering practicability of the passive positioning of two stations. A positioning system can both be independent of the length of the baseline and at the same time self-determine the length of the baseline. This characteristic will undoubtedly provide a mathematical basis for constructing a dynamic intelligent location system that can organize the whole detection process by itself.

\section{Derivation}

Figure 1 shows the two-station positioning system based on the mixed measurement method of azimuth and time difference using mixed coordinates. Assume the coordinate positions of the two receiving stations are $A\left(x_{a}, y_{a}\right)$ and $B\left(x_{b}, y_{b}\right)$ respectively, the target radiation source is located at $T(x, y)$, and the baseline length between the two stations is $d$.

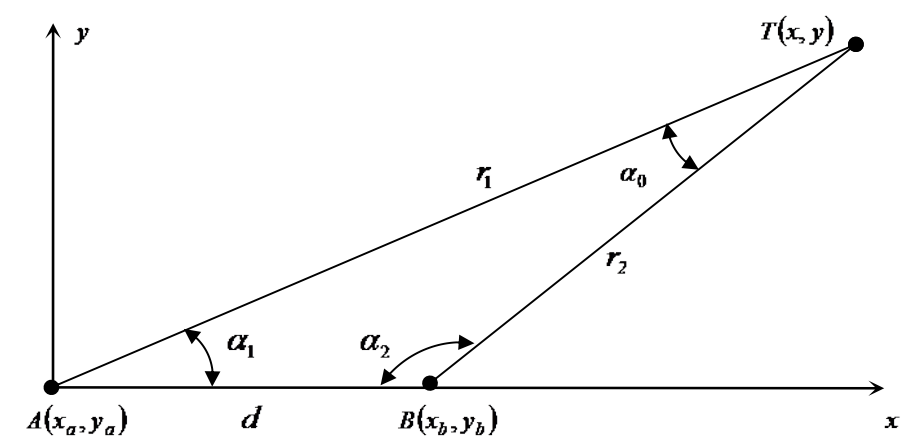

Figure 1. Geometric description of double station positioning system. 
Among them, the intersection angle $\alpha_{0}=180^{\circ}-\alpha_{1}-\alpha_{2}$ between the radial distance from the target to the two stations is not an independent parameter, but only a transitional parameter for mathematical derivation. Note that Figure 1 does not explicitly specify the origin of the coordinate system.

\subsection{Target Position in Polar Coordinates}

In many cases, direct mathematical analysis using polar coordinate systems will yield simpler mathematical expressions. At this point, if the coordinate origin is set at site A, i.e.: $x_{a}=0, y_{a}=0$, the derivation result will be more concise. According to the sine theorem

$$
\begin{aligned}
& \frac{r_{1}}{\sin \alpha_{2}}=\frac{d}{\sin \alpha_{0}} \\
& \frac{r_{2}}{\sin \alpha_{1}}=\frac{d}{\sin \alpha_{0}}
\end{aligned}
$$

where, $r_{i}$ is the radial distance; $\alpha_{i}$ the target azimuth angle of each station measured against the interstation baseline $(i=1,2)$.

By organizing, the following can be obtained

$$
\begin{aligned}
& r_{1}=\frac{d \sin \alpha_{2}}{\sin \alpha_{0}} \\
& r_{2}=\frac{d \sin \alpha_{1}}{\sin \alpha_{0}}
\end{aligned}
$$

Further based on the time difference measurement, there are

$$
\Delta r=v_{c} \Delta t=r_{1}-r_{2}
$$

where: $v_{c}$ is the speed of light; $\Delta t$ the time difference.

If Equations (3) and (4) are substituted into Equation (5), we can get

$$
v_{c} \Delta t=\frac{d}{\sin \alpha_{0}}\left(\sin \alpha_{2}-\sin \alpha_{1}\right)
$$

Using the results of Equation (1) again, we can get

$$
v_{c} \Delta t=\frac{r_{1}}{\sin \alpha_{2}}\left(\sin \alpha_{2}-\sin \alpha_{1}\right)
$$

From this, a ranging measurement formula is obtained, which is only related to the azimuth angle and time difference, but independent of the baseline length

$$
r_{1}=\frac{v_{c} \Delta t \sin \alpha_{2}}{\sin \alpha_{2}-\sin \alpha_{1}} .
$$

\subsection{The Target Position in Cartesian Coordinates}

According to the traditional mathematical analysis method, the equations are usually listed according to the positioning conditions. At this point, without loss of generality, let site $\mathrm{A}$ is not the origin of coordinates, namely: $x_{a} \neq 0, y_{a} \neq 0$. According to the direction-finding method, there are 


$$
\operatorname{tg} \alpha_{1}=\frac{y-y_{a}}{x-x_{a}}
$$

According to the measurement method of time difference:

$$
v_{c} \Delta t=\sqrt{\left(x_{a}-x\right)^{2}+\left(y_{a}-y\right)^{2}}-\sqrt{\left(x-x_{b}\right)^{2}+\left(y-y_{b}\right)^{2}}
$$

A solution independent of the baseline length can also be obtained by the joint solution of the equation. However, the existing analysis results have shown that [3], it is difficult to obtain the display solution directly in the Cartesian coordinate system, and on the other hand, there will be the problem of positioning ambiguity.

In fact, if coordinate transformation is carried out on the derivation result of polar coordinate system, the linear display solution can be obtained, and the general expression is

$$
\begin{gathered}
x-x_{a}=r_{1} \cos \alpha_{1}=\frac{v_{c} \Delta t \cos \alpha_{1} \sin \alpha_{2}}{\sin \alpha_{2}-\sin \alpha_{1}} \\
y-y_{b}=r_{1} \sin \alpha_{1}=\frac{v_{c} \Delta t \sin \alpha_{1} \sin \alpha_{2}}{\sin \alpha_{2}-\sin \alpha_{1}} .
\end{gathered}
$$

\subsection{Baseline Length between Two Stations}

Another feature of the two-station location based on azimuth and time difference is that the baseline length between two stations can also be taken as an unknown quantity and can be solved based only on the measurement of angle and time difference.

Based on the above derivation results, the baseline length between the two stations can be solved by using Equation (6)

$$
d=\frac{v_{c} \Delta t \sin \left(180^{\circ}-\alpha_{1}-\alpha_{2}\right)}{\sin \alpha_{2}-\sin \alpha_{1}} .
$$

\subsection{The Characteristics of Solution}

In fact, the linear display solution derived in this paper is universal and is not a particular solution that can only be used for localization without baseline. The obtained solution not only shows that the dual-station positioning system can be applied without baseline, but also can be used directly in the cases where the baseline length has been determined. In addition, compared with the existing research results [3] [4] [5], the results obtained in this paper are obviously more concise and therefore more applicable.

If you look at the solution equation listed in terms of the definite solution condition in the cartesian coordinate system, you can see that it does not actually contain the baseline length, which means that the characteristic of no baseline has been implied in the process of solving, but people have not paid attention to it before.

Different from the existing two-station positioning model, the mathematical 
solution derived from the path difference positioning condition based on the polar coordinate system in this paper will diverge when the two base angles of the positioning triangle are the same. This mathematical property may not be useful for engineering applications. It may be a future research task whether we can deduce the mathematical expression without divergence.

\section{Simulation Verification}

By specifying the baseline length $d$ and the radial distance $r_{1}$ at site $\mathrm{A}$, and considering the azimuth $\alpha_{1}$ at site $\mathrm{A}$ as a continuous variation, the remaining edge and angle parameters of the positioning triangle can be determined. This includes the azimuth $\alpha_{2}$ and radial distance $r_{2}$ at site $\mathrm{B}$.

On this basis, the path difference between the two stations can be obtained from the calculated radial distance $r_{2}$ and the specified radial distance $r_{1}$, and then the calculated path difference can be used to replace the path difference actually obtained from the time difference measurement. Finally, the calculated value of the radial distance is given by the two-station ranging formula

$$
r_{1}^{c}=\frac{\left(r_{1}-r_{2}\right) \sin \alpha_{2}}{\sin \alpha_{2}-\sin \alpha_{1}}
$$

where, superscript $c$ represents the calculated value of the radial distance at site A. Actually $r_{2}$ and $\alpha_{2}$ are also calculated, but for the sake of simplicity, it's not labeled with superscript $c$.

The relative calculation error can be obtained by comparing the calculated value with the theoretical value of the initially specified radial distance

$$
\varepsilon_{r}=100 * \frac{\left|r_{1}^{c}-r_{1}\right|}{r_{1}}
$$

Figure 2 shows the relative calculation error curve when the specified radial distance $r_{1}$ is $100 \mathrm{~km}$ and the baseline length $d$ is $10 \mathrm{~km}$ and the azimuth angle $\alpha_{1}$ changes continuously between 0 and 90 degrees. The simulation verification results show that the relative calculation error does not change significantly when taking different baselines. Therefore, Figure 2 only schematically shows a curve.

The correctness of the calculation formula of the baseline length can also be verified in basically the same way. In this case, the theoretical value of the specified baseline length is compared with the calculated value

$$
\varepsilon_{d}=100 * \frac{\left|d^{c}-d\right|}{d}
$$

where:

$$
d^{c}=\frac{\left(r_{1}-r_{2}\right) \sin \left(\alpha_{1}+\alpha_{2}\right)}{\sin \alpha_{2}-\sin \alpha_{1}}
$$

It must be noted in the simulation that for short baselines, when the azimuth at station $\mathrm{A}$ is acute, the azimuth at station $\mathrm{B}$ should be obtuse. 


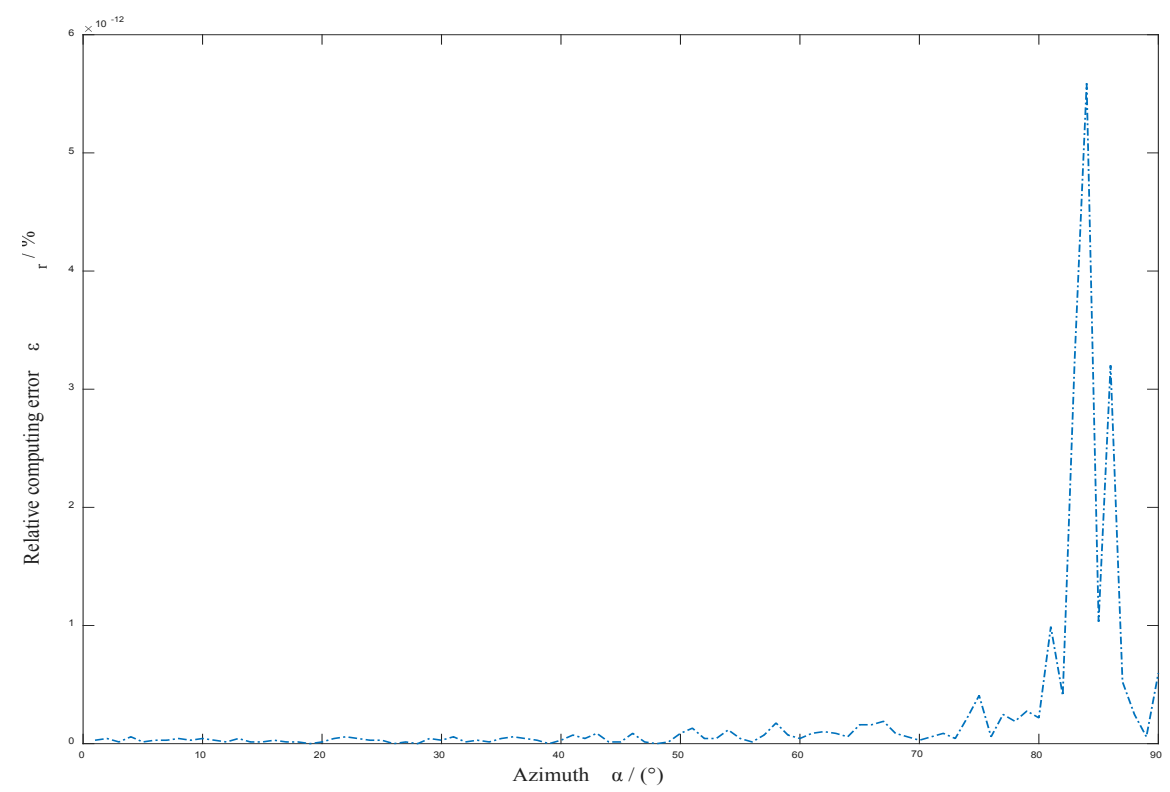

Figure 2. Relative calculation error curve.

\section{Error Analysis}

The total differential method is used to analyze the relative ranging error caused by the measurement error of time difference and angle. When the errors of each observation amount are zero mean, independent from each other, and the standard deviation is $\sigma_{\Delta t}$ and $\sigma_{\alpha}$, the relative ranging error formula is

$$
\frac{\mathrm{d} r_{1}}{r_{1}}=\frac{1}{r_{1}}\left(\left|\frac{\partial r_{1}}{\partial \Delta t}\right| \sigma_{\Delta t}+\left|\frac{\partial r_{1}}{\partial \alpha_{1}}\right| \sigma_{\alpha}+\left|\frac{\partial r_{1}}{\partial \alpha_{2}}\right| \sigma_{\alpha}\right)
$$

where, $\sigma_{\Delta t}$ and $\sigma_{\alpha}$ are respectively the root mean square value of the measurement error of time difference and azimuth angle.

The partial derivatives of radial distance with respect to each variable are

$$
\begin{aligned}
& \frac{\partial r_{1}}{\partial \Delta t}=\frac{v_{c} \sin \alpha_{2}}{\sin \alpha_{2}-\sin \alpha_{1}} \\
& \frac{\partial r_{1}}{\partial \alpha_{1}}=\frac{\Delta r \sin \alpha_{2} \cos \alpha_{1}}{\left(\sin \alpha_{2}-\sin \alpha_{1}\right)^{2}} \\
& \frac{\partial r_{1}}{\partial \alpha_{2}}=-\frac{\Delta r \sin \alpha_{1} \cos \alpha_{2}}{\left(\sin \alpha_{2}-\sin \alpha_{1}\right)^{2}}
\end{aligned}
$$

\subsection{The Effect of Baseline Length on Positioning Accuracy}

Treat the azimuth $\alpha_{1}$ at station A as a continuous change, Figure 3 shows the relative ranging error at different baseline lengths. Although the positioning formula does not include the baseline length, the positioning accuracy is clearly proportional to the baseline length. And the calculation shows that when the azimuth approaches zero, that is, when it approaches the direction of the baseline between the two stations, the singularity will appear. From the mathematical 


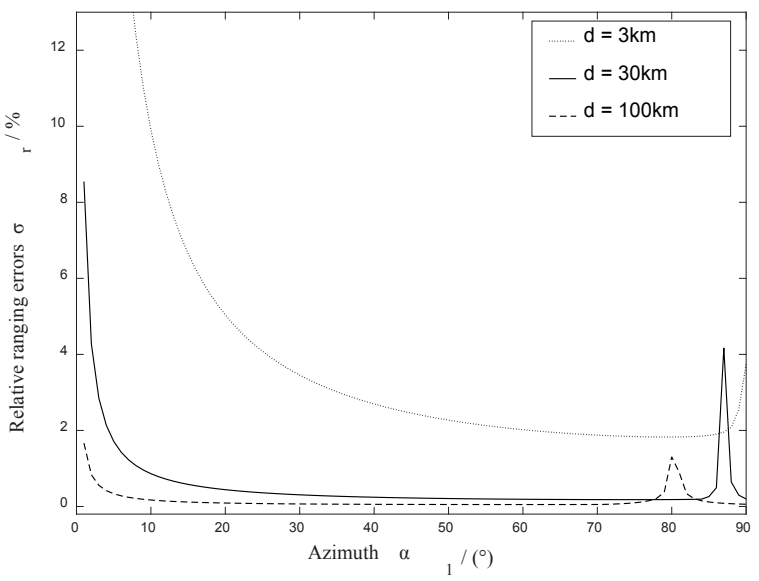

Figure 3. Relative ranging errors of different baseline lengths.

explanation, this is because in order to satisfy the sum of the interior angles of the triangle is equal to 180 degrees, when the azimuth of station A approaches 0 degrees, the azimuth of station $\mathrm{B}$ will have to approach 180 degrees. So the denominator of the error formula is going to zero.

For the same reason, unless the baseline is fairly long, as the azimuth of site A approaches 90 degrees, the azimuth of site B will follow as it approaches 90 degrees, so that the denominator of the error formula will approach 0 , resulting in divergence. However, by broadening the detection range, a shorter baseline can also meet the $5 \% \mathrm{R}$ technical requirement. For example, in an area with an azimuth of about 20 degrees or more, detection can be performed using a baseline of only 3 kilometers.

The root mean square value of measurement error of each variable used in error analysis is: $\sigma_{\Delta t}=100 \mathrm{~ns}, \sigma_{\alpha}=0.5^{\circ} \pi / 180^{\circ}$. Specify the radial distance: $r_{1}=300 \mathrm{~km}$.

\subsection{The Influence of Direction Finding Accuracy on Positioning Accuracy}

Treat the azimuth $\alpha_{1}$ at station $\mathrm{A}$ as a continuous change, Figure 4 shows the relative ranging error at different direction-finding accuracy. Obviously, the positioning accuracy is directly proportional to the accuracy of the measurement error RMS of direction-finding.

Divergence occurs at 90 degrees for the same reason as before. The RMS value of measurement error used in error analysis is: $\sigma_{\Delta t}=100 \mathrm{~ns}$. Specify the radial distance: $r_{1}=300 \mathrm{~km}, d=30 \mathrm{~km}$.

\subsection{The Influence of Intersection Angle on Positioning Accuracy}

Figure 5 shows the relative ranging error at different intersection angles. Divergence will occur when the base angles of the positioning triangle are the same.

During the simulation calculation, the azimuth at site B is calculated from the azimuth at site $\mathrm{A}$ and the specified intersection angle using the relationship of the sum of the angles inside the triangle. 


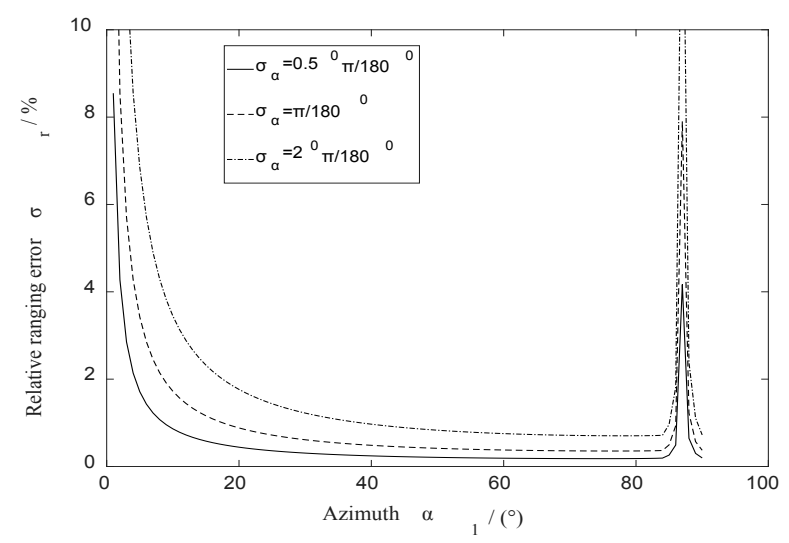

Figure 4. Relative ranging errors with different direction finding accuracy.

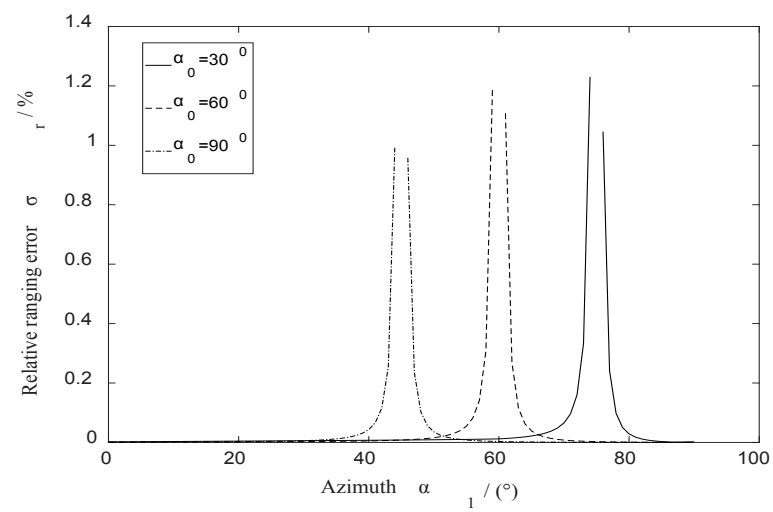

Figure 5. Influence of intersection angle on relative ranging error.

The RMS value of measurement error of each variable used in error analysis is: $\sigma_{\Delta t}=100 \mathrm{~ns}, \sigma_{\alpha}=0.5^{\circ} \pi / 180^{\circ}$. Specify the radial distance: $r_{1}=300 \mathrm{~km}$, $d=30 \mathrm{~km}$.

\subsection{The Effective Area That Can Be Co-Located}

The calculation shows that the relative ranging error of the positioning system is basically independent of the measurement accuracy of time difference.

In fact, starting directly from the linear solution, the advantage of using differential analysis is that you can directly observe the ranging errors at different target arrival angles [1]-[6]. Different from the pure angle dual station detection [7], the ranging error of dual-station positioning system based on the hybrid measurement of time difference and angle does not increase with the increase of target arrival angle, but is just the opposite.

According to the existing mathematical model, using a long baseline can avoid divergence, and it is possible to cover the whole area. In cases where short baselines have to be used, detection operation must be avoided at the midperpendicular between stations and at the vertical line at the site location. 


\section{Conclusions}

Based on the physical measurement, the main reason for obtaining the positioning solution without baseline is that the positioning conditions of the positioning system are actually redundant, which can be described as taking advantage of the redundant measurements of time difference or angle to replace the measurement of baseline length.

The research results of this paper provide a design basis for the dual-station passive positioning system in real-time maneuvering operation. In the case that the positioning solution is related to the baseline, a detection system that requires a long baseline cannot be operated flexibly without the auxiliary baseline measurement system. When the auxiliary measurement system is needed, it is often the time when the auxiliary measurement system fails [8]. The methods that do not require baseline measurements provide a more advantageous solution for their own real-time maneuvering detection platforms, such as airborne systems.

In the actual detection process, the detection accuracy of the two-station positioning system is closely related to the relative position between the detection station and the target. For a two-station positioning system, the available detection area is limited. One way to improve location accuracy is to add probe sites and extend probe areas. In fact, another method available is to follow the target and adjust the position of the probe station in real time. This introduces a concept of constructing intelligent dynamic positioning system. The research results of this paper also provide a design basis for intelligent positioning systems that need self-organizing capabilities [9]. Such an intelligent positioning system will be able to operate on its own without the technical support of other positioning systems, such as satellite positioning systems. In the real-time detection process, if the signal of the detected target is relatively stable, the two detection stations can simultaneously detect the relative position between the two stations while detecting the target position. With the help of a third party radiation source, the detection system can realize self-detection of the relative positions between the detection sites.

\section{Conflicts of Interest}

The author declares no conflicts of interest regarding the publication of this paper.

\section{References}

[1] Liu, C.F. (2011) Passive Location and Tracking. Xidian University Press, Xi'an.

[2] Wang, B.-C., He, Y., Wang, G.-H. and Du, C.-M. (2012) Related Issues in DualSensor Bearing-Only Localization. Opto-Electronic Engineering, 39, 80-87.

[3] Chen, L. and Li, S.H. (2003) Study on Passive DOA Jointed TOA Location Algorithm. Journal of Electronics \& Information Technology, 25, 771-776.

[4] Cui, Y. and Wang, Y.-L. (2011) Accuracy Analysis of Two Station DOA/TDOA Lo- 
cation. Radio Communications Technology, 37, 44-46, 57.

[5] Wang, Y.T. (2015) A Method of Passive AOA/TDOA Location Based on the Cosine Law. Modern Radar, 37, 41-46.

[6] Poisel, R.A. (2005) Electronic Warfare Target Location Methods. 2nd Edition, Artech House, London.

[7] Yu, T. (2017) Technology of Passive Detection Location. National Defense Industry Press, Beijing.

[8] Gao, S.S., Tao, Z. and Huang, Y. (2019) Construction Method of Mobile Ground Station Location System Based on Optimizing Algorithms. Laser Journal, 40, 114-117. https://doi.org/10.1088/1742-6596/1314/1/012158

[9] Zhu, W.Q., Wang, K.R. and Zhu, X.D. (2016) Key Techniques of Electronic Reconnaissance and Location Based on Micro/Nano Satellite Network. Aerospace Electronic Warfare, 32, 1-5. 